\title{
TEMPERATURE AND VELOCITY RELAXATION IN PLASMA. SPECTRAL THEORY APPROACH
}

\author{
S.A. Sokolovsky ${ }^{1}$, A.I. Sokolovsky²*, I.S. Kravchuk², O.A. Grinishin ${ }^{2}$ \\ ${ }^{1}$ Prydniprovska State Academy of Civil Engineering and Architecture, Dnipro, Ukraine \\ ${ }^{2}$ Oles Honchar Dnipro National University, Dnipro, Ukraine \\ *e-mail: alexander.i.sokolovsky@gmail.com
}

\begin{abstract}
The electron temperature and velocity relaxation of completely ionized plasma is studied on the basis of kinetic equation obtained from the Landau equation in a generalized Lorentz model. In this model contrary to the standard one ions form an equilibrium subsystem. Relaxation processes in the system are studied on the basis of spectral theory of the collision integral operator. This leads to an exact theory of relaxation processes of component temperatures and velocities equalizing. The relation of the developed theory with the Bogolyubov method of the reduced description of nonequilibrium systems is established, because the theory contains a proof of the relevant functional hypothesis, the idea of which is the basis of the Bogolyubov method. The temperature and velocity relaxation coefficients as eigenvalues of the collision integral operator are calculated by the method of truncated expansion of its eigenfunctions in the Sonine orthogonal polynomials. The coefficients are found in one- and two-polynomial approximation. As one can expect, convergence of this expansion is slow.

Keywords: plasma, generalized Lorentz model, relaxation coefficients, collision integral operator, spectral theory, one- and two-polynomial approximations, functional hypothesis.
\end{abstract}

Received 10.11.2019; Received in revised form 115.12.2019; Accepted 23.12.2019

\section{Introduction}

This paper is devoted to the study of relaxation processes in completely ionized plasma. The plasma is considered in the generalized Lorentz model, in which the ion subsystem is assumed to be in equilibrium. The model is based on the Landau kinetic equation [1].

For the first time the problem of equalizing the component temperatures in a system was considered by Landau [1] (the velocity relaxation was studied analogously in [2]). His research was based on the mentioned kinetic equation [1] (see also [3]). In his investigation he assumed without proof that the plasma components quickly become equilibrium and are described by the Maxwell distribution functions. A related problem in polaron theory was considered by Bogolyubov and Bogolyubov (Jr.) in book [4] where solution of the kinetic equation for a polaron were discussed with the same assumption about the Maxwell distribution. Our paper avoids this assumption.

Contrary to our papers [5-7], which are based on the Bogolyubov reduced description method [8] (see also a review in book [3]), the present paper develops kinetics of the system through elaborating the spectral theory of the collision integral operator.

The paper is constructed as it follows. In the Section 2 the generalized Lorentz model is formulated following to [7]. The Section 3 discusses the spectral theory of the collision integral operator which is used to solve the kinetic equation of the theory. The Section 4 describes the method of truncated expansion in the Sonine polynomials for the spectral problem solution. Section 5 presents calculation of the relaxation coefficients in one- and twopolynomial approximation.

\section{The generalized Lorentz model of plasma}

Completely ionized electron-ion plasma is investigated. The Landau kinetic equation lies in the basis of our consideration (see, for example, $[1,3]$ ). It is assumed that ions form an equilibrium system in a state of rest with temperature $T_{0}$ which are described by the Maxwell distribution 


$$
w_{i p} \equiv \frac{n_{0}}{\left(2 \pi M T_{0}\right)^{3 / 2}} e^{-\frac{p^{2}}{2 M T_{0}}}
$$

( $n_{0}$ is ion density, $M$ is an ion mass). Moreover, here ion-ion and electron-electron interactions are neglected. So, the investigation is based on the generalized Lorentz model [7]. Electron distribution function is denoted here by $f_{p}(x, t)$ and is normalized by the condition

$$
\int d^{3} p f_{p}(x, t)=n(x, t)
$$

where $n(x, t)$ is density of number of electrons. The kinetic equation for this function takes the form

$$
\partial_{t} f_{p}(x, t)=-\frac{p_{n}}{m} \frac{\partial f_{p}(x, t)}{\partial x_{n}}+I_{p}(f(x, t))
$$

where the ion-electron collision integral is given by the formula [7]

$$
I_{p}(f)=\frac{\partial}{\partial p_{n}}\left(D_{n l}(p)\left(\frac{\partial f_{p}}{\partial p_{l}}+\frac{p_{l}}{m T_{0}} f_{p}\right)\right)
$$

with function $D_{n l}(p)$ defined by the formula

$$
D_{n l}(p)=C \int d^{3} p^{\prime} w_{i p^{\prime}} S_{n l}\left(\frac{p}{m}-\frac{p^{\prime}}{M}\right), \quad C \equiv 2 \pi e^{4} z^{2} L
$$

( $m$ is an electron mass, $e z$ is an ion charge, $L$ is the Coulomb logarithm) where

$$
S_{n l}(u) \equiv\left(u^{2} \delta_{n l}-u_{n} u_{l}\right) / u^{3}
$$

Equilibrium electron distribution function $f_{p}$ is given by the Maxwell distribution

$$
f_{p}^{e q}=w_{p}, \quad w_{p} \equiv \frac{n}{\left(2 \pi m T_{0}\right)^{3 / 2}} e^{-\frac{p^{2}}{2 m T_{0}}} ; \quad I_{p}(w)=0 .
$$

The function $D_{n l}(p)$ defined in (5) has a fairly simple structure, which simplifies our further research. In particular, it has a simple dependence on dimensional quantities. This allows finding out the structure of dependence on the dimensional values of all objects of the theory.

\section{Dynamics of spatially uniform states of plasma}

In this paper we will limit ourselves to research of spatially uniform states of the system, for which distribution function $f_{p}(x, t)$ does not depend on coordinates $x_{n}$. Let us introduce collision operator $\hat{K}$ by the definition

$$
I_{p}(w \mathrm{f})=-w_{p} \hat{K} f_{p}
$$


( $\mathrm{f}_{p}$ is an arbitrary function). It is symmetric and positively defined one at the definition of a scalar product by the formula

$$
\left(\mathrm{f}_{1}, \mathrm{f}_{2}\right)=\int d^{3} p w_{p} \mathrm{f}_{1 p} \mathrm{f}_{2 p} .
$$

Therefore its eigenfunctions $g_{i p}$ and the corresponding eigenvalues $\lambda_{i}$

$$
\hat{K} g_{i p}=\lambda_{i} g_{i p}
$$

have the properties

$$
\lambda_{i}>0, \quad\left(g_{i}, g_{i^{\prime}}\right) \equiv b_{i} \delta_{i i^{\prime}}
$$

( $b_{i}$ are some values). Note, that the spectral theory of the collision integral operator $\hat{K}$ is used too in our paper [9] for the investigation of electron mobility in plasma and in paper [10] for the polaron theory in semiconductors.

Let us find a solution of (3) in the form

$$
f_{p}=w_{p}\left(1+g_{p}\right) .
$$

The function $g_{p}$ is normalized by the definition

$$
\left\langle g_{p}\right\rangle=0
$$

where the average value notation with the distribution $w_{p}$ is introduced

$$
\left\langle\mathrm{f}_{p}\right\rangle=\int d^{3} p w_{p} \mathrm{f}_{p} .
$$

Kinetic equation (3) takes the form

$$
\partial_{t} g_{p}=-\hat{K} g_{p}
$$

and can be solved in terms of eigenfunctions $g_{i p}$ and eigenvalues $\lambda_{i}$ of the operator $\hat{K}$

$$
g_{p}=\sum_{i} c_{i} g_{i p} e^{-\lambda_{i} t}
$$

with some coefficients defined by the initial condition for the distribution function $f_{p}(t=0) \equiv f_{p 0}$. Eigenvalues $\lambda_{i}$ define relaxation times $\tau_{i} \equiv \lambda_{i}^{-1}$ of the system. According to (13) and (16), average values of the eigenfunctions are equal to zero

$$
\left\langle g_{i p}\right\rangle=0 .
$$

Let us study the evolution of the electron subsystem in terms of momentum $\pi_{n}$ and energy $\varepsilon$ densities

$$
\pi_{n} \equiv \int d^{3} p f_{p} p_{n}=\left\langle g_{p} p_{n}\right\rangle, \quad \varepsilon \equiv \int d^{3} p \varepsilon_{p} f_{p}=\left\langle g_{p} \varepsilon_{p}\right\rangle+\varepsilon_{0}, \quad \varepsilon_{0} \equiv \frac{3}{2} n T_{0} .
$$

Among eigenfunctions $g_{i p}$ there are vector $A_{p} p_{n}$ and scalar $B_{p}$ ones with the corresponding eigenvalues $\lambda_{u}, \lambda_{T}$ 


$$
\hat{K} A_{p} p_{l}=\lambda_{u} A_{p} p_{l}, \quad \hat{K} B_{p}=\lambda_{T} B_{p}
$$

i.e. $A_{p}, B_{p}$ are scalar functions of $p$. Quantities $A_{p}, B_{p}$ are defined by (19) with an accuracy up to arbitrary factors. Let us choose additional conditions for equations (19) to eliminate this uncertainty

$$
\left\langle A_{p} \varepsilon_{p}\right\rangle=3 n / 2, \quad\left\langle B_{p} \varepsilon_{p}\right\rangle=3 n / 2, \quad\left\langle B_{p}\right\rangle=0
$$

(the last relation is added according to (17)). Therefore expression (16) for $g_{p}$ can be rewritten in the form

$$
g_{p}=c_{n} A_{p} p_{n} e^{-\lambda_{u} t}+c B_{p} e^{-\lambda_{T} t}+\sum_{i^{\prime}} c_{i^{\prime}} g_{i^{\prime} p} e^{-\lambda_{i^{\prime}} t}
$$

where subs $i^{\prime}$ enumerate other eigenfunctions and eigenvalues.

Let us assume that relaxation times of the system satisfy the condition that some time $\tau_{0}$ exits with the property

$$
\tau_{T}, \tau_{u} \gg \tau_{0}>\tau_{i^{\prime}} .
$$

Then relation (21) at $t \gg \tau_{0}$ takes the form

$$
g_{p} \underset{t>>\tau_{0}}{\longrightarrow} g_{p}^{(+)} \equiv c_{n} A_{p} p_{n} e^{-\lambda_{u} t}+c B_{p} e^{-\lambda_{T} t} .
$$

According to (18), (20) at these times momentum $\pi_{n}$ and energy $\varepsilon$ densities are given by relations

$$
\pi_{n} \underset{t>>\tau_{0}}{\longrightarrow} \pi_{n}^{(+)} \equiv m n c_{n} e^{-\lambda_{u} t}, \quad \varepsilon \underset{t>>\tau_{0}}{\longrightarrow} \varepsilon^{(+)} \equiv \frac{3 n}{2} c e^{-\lambda_{T} t}+\varepsilon_{0}
$$

and therefore asymptotic function $g_{p}^{(+)}$can be written in the form

$$
g_{p}^{(+)} \equiv \pi_{n}^{(+)} A_{p} p_{n} / m n+\left(\varepsilon^{(+)}-\varepsilon_{0}\right) B_{p} 2 / 3 n .
$$

Variables $\varepsilon^{(+)}, \pi_{n}^{(+)}$satisfy the time equations

$$
\partial_{t} \varepsilon^{(+)}=-\lambda_{T}\left(\varepsilon^{(+)}-\varepsilon_{0}\right), \quad \partial_{t} \pi_{n}^{(+)}=-\lambda_{u} \pi_{n}^{(+)}
$$

and describe relaxation processes in the electron subsystem. Let us introduce as observable variables instead of $\varepsilon^{(+)}, \pi_{n}^{(+)}$electron subsystem temperature $T$ and mass velocity $u_{n}$

$$
\varepsilon^{(+)}=\left(3 n T+m n u^{2}\right) / 2, \quad \pi_{n}^{(+)}=m n u_{n} .
$$

These variables satisfy the relaxation equations

$$
\partial_{t} u_{n}=-\lambda_{u} u_{n}, \quad \partial_{t} T=-\lambda_{T}\left(T-T_{0}\right)+\left(2 \lambda_{u}-\lambda_{T}\right) m u^{2} / 3
$$

and asymptotic electron distribution function takes the form

$$
g_{p}^{(+)}=u_{n} A_{p} p_{n}+\left(T-T_{0}+m u^{2} / 3\right) B_{p} .
$$


In fact this formula expresses the idea of the Bogolyubov functional hypothesis [8] that is the basis of his method of the reduced description of nonequilibrium processes (see a review in [3]). So, the theory developed in the present paper contains a proof of this hypothesis for the case under consideration.

\section{Method of the truncated expansion in the Sonine polynomials for spectral problem solution}

Here at approximate solution of equations (19) by an expansion in orthogonal polynomials the Sonine polynomials $S_{q}^{\alpha}(x)(q=0,1,2, \ldots, \alpha$ is a real number $)$ are used (see, for example, $[6,7,9]$ ). Let us find the functions $A_{p}, B_{p}$ in the form of expansions

$$
A_{p}=\sum_{q=0}^{\infty} a_{q} S_{q}^{3 / 2}\left(\beta \varepsilon_{p}\right), \quad \quad B_{p}=\sum_{q=0}^{\infty} b_{q} S_{q}^{1 / 2}\left(\beta \varepsilon_{p}\right) \quad\left(\beta \equiv T_{0}^{-1}\right)
$$

The equations for the coefficients $a_{q}$ and $b_{q}$ are found by the next way. Substituting the expansions (30) into equations (19), multiplying them by $p_{l} S_{q}^{3 / 2}\left(\beta \varepsilon_{p}\right)$ and $S_{q}^{1 / 2}\left(\beta \varepsilon_{p}\right)$, correspondingly, and taking the average value with the distribution function $w_{p}$ in the both sides gives

$$
\sum_{q^{\prime}=0}^{\infty} A_{q q^{\prime}} a_{q^{\prime}}=\lambda_{u} a_{q} x_{q}, \quad \quad \sum_{q^{\prime}=0}^{\infty} B_{q q^{\prime}} b_{q^{\prime}}=\lambda_{T} b_{q} y_{q} .
$$

Here the notations

$$
\begin{array}{cc}
A_{q q^{\prime}}=\left\{p_{l} S_{q}^{3 / 2}\left(\beta \varepsilon_{p}\right), p_{l} S_{q^{3}}^{3 / 2}\left(\beta \varepsilon_{p}\right)\right\}, & B_{q q^{\prime}}=\left\{S_{q}^{1 / 2}\left(\beta \varepsilon_{p}\right), S_{q^{\prime}}^{1 / 2}\left(\beta \varepsilon_{p}\right)\right\} \\
x_{q} \equiv \frac{4 m n}{\beta} \frac{\Gamma(q+5 / 2)}{\pi^{1 / 2} q !}, & y_{q} \equiv 2 n \frac{\Gamma(q+3 / 2)}{\pi^{1 / 2} q !}
\end{array}
$$

are introduced and the bilinear forms (brackets) defined by the formula [7]

$$
\left\{\mathrm{f}_{1 p}, \mathrm{f}_{2 p}\right\}=\int d^{3} p w_{p} \mathrm{f}_{1 p} \hat{K} \mathrm{f}_{2 p}=\int d^{3} p w_{p} D_{n l}(p) \frac{\partial \mathrm{f}_{1 p}}{\partial p_{n}} \frac{\partial \mathrm{f}_{2 p}}{\partial p_{l}}
$$

are used $\left(\mathrm{f}_{1 p}\right.$ and $\mathrm{f}_{2 p}$ are arbitrary functions).

Additional conditions (20) give the contributions of the first polynomials to expansion (30)

$$
a_{0}=\beta ; \quad b_{0}=0, \quad b_{1}=-\beta .
$$

We will say that the solutions of equations (31) are calculated in the $s$-polynomial approximation if all the coefficients of the expansions (30) starting from $(s+1)$-th are assumed to be zero: $a_{q}^{\left[s_{1}\right]}=0(q=0,1,2, \ldots)$ at $q \geq s, b_{q}^{\left[s_{1}\right]}=0(q=1,2, \ldots)$ at $q \geq s+1$ and $a_{0}^{[s]}=\beta, b_{1}^{[s]}=-\beta(s=1,2, \ldots)$. Hereafter index $s$ at a quantity $a^{[s]}$ indicates the number of polynomials used in the considered approximation. At the same time the obtained from (31) equations give the corresponding expressions $\lambda_{u}^{[s]}, \lambda_{T}^{[s]}$ for relaxation coefficients.

The calculations in the one- and two-polynomial approximations give [7] 


$$
\begin{gathered}
\lambda_{u}^{[1]}=A_{00} / x_{0}, \quad \lambda_{T}^{[1]}=B_{11} / y_{1} ; \\
\left.\lambda_{u}^{[2]}=\left\{\left(A_{11}+\frac{x_{1}}{x_{0}} A_{00}\right)-\left[\left(A_{11}-\frac{x_{1}}{x_{0}} A_{00}\right)^{2}+4 \frac{x_{1}}{x_{0}} A_{01}^{2}\right)\right]^{1 / 2}\right\} \frac{1}{2 x_{1}}, \\
\left.\lambda_{T}^{[2]}=\left\{\left(B_{22}+\frac{y_{2}}{y_{1}} B_{11}\right)-\left[\left(B_{22}-\frac{y_{2}}{y_{1}} B_{11}\right)^{2}+4 \frac{y_{2}}{y_{1}} B_{12}^{2}\right)\right]^{1 / 2}\right\} \frac{1}{2 y_{2}} .
\end{gathered}
$$

Coefficients $\lambda_{u}^{[2]}, \lambda_{T}^{[2]}$ satisfy quadratic equations and in (35) the smallest roots of this equations are given which define the slowest evolution of the system.

A detailed analysis of the next many-polynomial approximations is very cumbersome and can be conducted in some limiting cases [11].

\section{Calculation of the relaxation coefficients $\lambda_{u}, \lambda_{T}$ \\ in one- and two-polynomial approximation}

According to (32) we have to calculate the following functions $A_{q q^{\prime}}$ and $B_{q q^{\prime}}$

$$
\begin{gathered}
A_{00}=\left\{p_{l}, p_{l}\right\}, \quad A_{01}=\frac{5}{2}\left\{p_{l}, p_{l}\right\}-\beta\left\{p_{l}, \varepsilon_{p} p_{l}\right\}, \\
A_{11}=\frac{25}{4}\left\{p_{l}, p_{l}\right\}-5 \beta\left\{p_{l}, \varepsilon_{p} p_{l}\right\}+\beta^{2}\left\{\varepsilon_{p} p_{l}, \varepsilon_{p} p_{l}\right\} ; \\
B_{11}=\beta^{2}\left\{\varepsilon_{p}, \varepsilon_{p}\right\}, \quad B_{12}=-\frac{1}{2} \beta^{3}\left\{\varepsilon_{p}, \varepsilon_{p}^{2}\right\}+\frac{5}{2} \beta^{2}\left\{\varepsilon_{p}, \varepsilon_{p}\right\}, \\
B_{22}=\frac{1}{4} \beta^{4}\left\{\varepsilon_{p}^{2}, \varepsilon_{p}^{2}\right\}-\frac{5}{2} \beta^{3}\left\{\varepsilon_{p}^{2}, \varepsilon_{p}\right\}+\frac{25}{4} \beta^{2}\left\{\varepsilon_{p}, \varepsilon_{p}\right\} .
\end{gathered}
$$

In our paper [7] the formula for calculation of the brackets (33) was obtained

$$
\left\{a_{p}, b_{p}\right\}=\left.\frac{n n_{0} m^{1 / 2} C}{\left(1+\mu^{2}\right)^{1 / 2} T_{0}^{1 / 2}} \int d^{3} q_{c} d^{3} q w_{q_{c}} w_{q} S_{n l}(q) \frac{\partial a_{p}}{\partial p_{n}} \frac{\partial b_{p}}{\partial p_{l}}\right|_{p \rightarrow \frac{\left(m T_{0}\right)^{1 / 2}}{\left(1+\mu^{2}\right)^{1 / 2}}\left(\mu q_{c}+q\right)}
$$

where

$$
w_{q} \equiv \frac{1}{(2 \pi)^{3 / 2}} e^{-\frac{q^{2}}{2}}, \quad \mu \equiv\left(\frac{m}{M}\right)^{1 / 2} .
$$

At calculation of the brackets with the formula (37) is convenient at first to take integrals over $q_{c n}$ and then ones over $q_{n}$. By this way we obtain

$$
\begin{aligned}
\left\{p_{s}, p_{s}\right\} & =\frac{m n T_{0}}{\left(1+\mu^{2}\right)^{1 / 2}} \lambda, \quad\left\{p_{s}, \varepsilon_{p} p_{s}\right\}=\frac{m n T_{0}^{2}\left(1+4 \mu^{2}\right)}{2\left(1+\mu^{2}\right)^{3 / 2}} \lambda, \\
\left\{\varepsilon_{p} p_{s}, \varepsilon_{p} p_{s}\right\} & =\frac{m n T_{0}^{3}\left(55 \mu^{4}+36 \mu^{2}+8\right)}{4\left(1+\mu^{2}\right)^{5 / 2}} \lambda ; \quad\left\{\varepsilon_{p}, \varepsilon_{p}\right\}=\frac{n T_{0}^{2} \mu^{2}}{\left(1+\mu^{2}\right)^{3 / 2}} \lambda,
\end{aligned}
$$




$$
\left\{\varepsilon_{p}^{2}, \varepsilon_{p}\right\}=\frac{n T_{0}^{3} \mu^{2}\left(5 \mu^{2}+2\right)}{\left(1+\mu^{2}\right)^{5 / 2}} \lambda, \quad\left\{\varepsilon_{p}^{2}, \varepsilon_{p}^{2}\right\}=\frac{n T_{0}^{4} \mu^{2}\left(35 \mu^{4}+28 \mu^{2}+8\right)}{\left(1+\mu^{2}\right)^{7 / 2}} \lambda
$$

where

$$
\lambda \equiv \frac{n_{0} 2^{3 / 2} C}{\pi^{1 / 2} m^{1 / 2} T_{0}^{3 / 2}} .
$$

In two-polynomial approximation relations (39) give a very cumbersome results. Therefore, here the relaxation constants $\lambda_{T}$ and $\lambda_{u}$ are calculated at $\mu=1$ that corresponds to the physical sense of $\mu=(m / M)^{1 / 2}$ where $m$ and $M$ are electron and ion masses. Therefore, all values in (36) are taken with the same accuracy only in the first two approximations

$$
\begin{aligned}
& A_{00} \approx \lambda m n T_{0}\left(1-\frac{1}{2} \mu^{2}\right), \quad A_{01} \approx \lambda m n T_{0} 2\left(1-\frac{5}{4} \mu^{2}\right), \quad A_{11} \approx \lambda m T_{0} \frac{1}{4}\left(23-\frac{43}{2} \mu^{2}\right), \\
& B_{11} \approx \lambda n \mu^{2}\left(1-\frac{3}{2} \mu^{2}\right), \quad B_{12} \approx \lambda n \mu^{2} \frac{3}{2}\left(1+\frac{5}{2} \mu^{2}\right), \quad B_{22} \approx \lambda n \mu^{2} \frac{1}{4}\left(13-\frac{75}{2} \mu^{2}\right) .
\end{aligned}
$$

Using formulas (35) gives the relaxation coefficients $\lambda_{T}$ and $\lambda_{u}$ in one- and twopolynomial approximations with the corresponding accuracy

$$
\begin{gathered}
\lambda_{u}^{[1]} \approx \lambda \frac{1}{3}\left(1-\frac{1}{2} \mu^{2}\right), \quad \lambda_{T}^{[1]} \approx \lambda \mu^{2} \frac{2}{3}\left(1-\frac{3}{2} \mu^{2}\right) \\
\lambda_{u}^{[2]} \approx \lambda \frac{1}{4}\left[\left(33-809^{1 / 2}\right)+\frac{1}{2}\left(-53+\frac{2029}{809^{1 / 2}}\right) \mu^{2}\right] \frac{1}{15} \approx \lambda\left(0,076+0,153 \mu^{2}\right), \\
\lambda_{T}^{[2]} \approx \lambda \mu^{2}\left[\frac{1}{2}\left(9-61^{1 / 2}\right)+\left(-\frac{45}{4}+\frac{345}{61^{1 / 2}}\right) \mu^{2}\right] \frac{4}{15} \approx \lambda \mu^{2}\left(0,484+14,0 \mu^{2}\right)
\end{gathered}
$$

These formulas allow comparing of both approximations

$$
\frac{\lambda_{u}^{[2]}-\lambda_{u}^{[1]}}{\lambda_{u}^{[1]}} \approx-0,77+0,83 \mu^{2}, \quad \frac{\lambda_{T}^{[2]}-\lambda_{T}^{[1]}}{\lambda_{T}^{[1]}} \approx-0,484+14,0 \mu^{2}
$$

This result shows that the convergence of the method of expansion in orthogonal Sonine polynomials used by us is at least very slow. There is no argument to expect the convergence of the procedure for the spectral problem because the problem is equivalent to solving the Fredholm integral equation of the second kind (see, for example, [12]).

\section{Conclusions}

The paper investigates completely ionized plasma in the generalized Lorentz model, which considers the system of ions to be equilibrium one and neglect electron-electron and ion-ion interactions. Component temperature and velocity relaxations are studied in spatially uniform states of the plasma. The investigation is based on the spectral theory of the collision integral operator that is linear one. Relaxation processes are studied without assumption that they are near their completion. The tensor dimensionality of the eigenfunctions of the collision integral operator determines the sense of parameters that describe the plasma evolution. Electron temperature and velocity relaxations are 
described by scalar and vector eigenfunctions. Relevant spectral problem is related to the Fredholm integral equations of the second kind. This problem is solved in the paper by the method of truncated expansion of the eigenfunctions in the Sonine orthogonal polynomials. The convergence of this method cannot by proved. Therefore, we calculate relaxation coefficients (eigenfunctions) in one- and two-polynomial approximations and compare them. To avoid cumbersome calculations our investigation is conducted in the limit of small electron-to-ion mass ratio with accuracy up to the first order after the leading contribution included. In is shown that the convergence of the expansion in the Sonine polynomials is at least very slow.

\section{References}

1. Landau, L.D. Kineticheskoe uravnenie v sluchae kulonovskogo vzaimodejstviya / L.D. Landau // ZhETF. - 1936. - Vol. 7. - P. 203 - 209 (in Russian).

2. Alexandrov, A.F. Osnovy elekrodinamiki plazmy /A.F. Aleksandrov, L.S. Bogdankevich, A.A. Rukhadze // Moscow: Vysshaya shkola. - 1988. - 424 p. (in Russian).

3. Akhiezer, A.I. Methods of Statistical Physics/ A.I. Akhiezer, S.V. Peletminskii. Amsterdam: Elsevier. - 2013. - 446 p.

4. Bogolyubov, N.N. Aspekty teorii polyarona / N.N. Bogolyubov, N.N. Bogolyubov (Jr.). - Moscow: Fizmatlit, 2004. - 175 p. (in Russian).

5. Sokolovsky, S.A. Relaksatsionnye yavleniya dlya elektrona $v$ kristalle i obobshchenie metoda Chepmena-Enskoga / S.A. Sokolovsky, A.I. Sokolovsky, I.M. Chernenko // Visnyk Kharkivskogo Universytetu, Fizychna seriya "Yadra, chastynky, polya". - 2004. - Vol. 642, Iss. 3(25). - P. 84 - 88 (in Russian).

6. Sokolovsky, S.A. Toward polaron kinetics in the Bogolyubov reduced description method / S.A. Sokolovsky // Theoretical and Mathematical Physics. - 2011. - Vol. 168, No. 2. - P. $1150-1164$.

7. Sokolovsky, S.A. Relaxation processes in completely ionized plasma in generalized Lorentz model / S.A. Sokolovsky, A.I. Sokolovsky, I.S. Kravchuk, O.A. Grinishyn // Journal of Physics and Electronics. - 2018. - Vol. 26(2). - P. 17 - 28.

8. Bogolyubov, N.N. Problems of a Dynamical Theory in Statistical Physics. Studies in Statistical Mechanics / N.N. Bogolyubov. - Amsterdam: North-Holland, 1962. $-130 \mathrm{p}$.

9. Sokolovsky, S. Mobility of electrons in plasma / S. Sokolovsky, A. Sokolovsky // 2019 IEEE 2nd Ukraine Conference on Electrical and Computer Engineering (UKRCON2019) (July 2-6, 2019, Lviv, Ukraine) Proceedings. - P. 783 - 787. ISBN: 978-1-72813882-4.

10. Sokolovsky, S.A. On theory of nonlinear relaxation in polaron subsystem of polar semiconductors / S.A. Sokolovsky // Proceedings of XXII ${ }^{\text {nd }}$ International Seminar/Workshop on direct and inverse problems of electromagnetic and acoustic wave theory (DIPED) (September 25-28, 2017, Dnipro, Ukraine). - 2017. - P. 242 - 247.

11. Gorev, V.N. Hydrodynamic, kinetic modes of plasma and relaxation damping of plasma oscillations / V.N. Gorev, A.I. Sokolovsky // Ukrainian Journal of Physics. 2015. - Vol. 60, No. 3. - P. 232 - 246.

12. Polyanin, A.D. Handbook of Integral Equations / A.D. Polyanin, A.V. Manzhirov. - Taylor\&Francis, 2008. - 1108 p. 